\title{
Surgically-Created Resection Cavity
}

National Cancer Institute

\section{Source}

National Cancer Institute. Surgically-Created Resection Cavity. NCI Thesaurus. Code C20051.

The space that is left after the surgical removal of a body part or tumor. 